\title{
G-Protein-coupled Estrogen Receptor 1 Agonist G-1 Perturbs Sunitinib Resistance-related Phosphoproteomic Signatures in Renal Cell Carcinoma
}

\author{
SHAO-KUAN CHEN ${ }^{1,2}$, YEN-CHIEH WANG ${ }^{1,3}$, TAI-YUAN LIN ${ }^{1}$, \\ HSIN-JOU WU ${ }^{1}$, CHI-JUNG HUANG ${ }^{4,5}$ and WEI-CHI KU ${ }^{1}$ \\ ${ }^{1}$ School of Medicine, College of Medicine, Fu Jen Catholic University, New Taipei City, Taiwan, R.O.C.; \\ ${ }^{2}$ Department of Surgery, Sijhih Cathay General Hospital, New Taipei City, Taiwan, R.O.C.; \\ ${ }^{3}$ Division of Urology, Cathay General Hospital, Taipei City, Taiwan, R.O.C.; \\ ${ }^{4}$ Department of Medical Research, Cathay General Hospital, Taipei City, Taiwan, R.O.C.; \\ ${ }^{5}$ Department of Biochemistry, National Defense Medical Center, Taipei City, Taiwan, R.O.C.
}

\begin{abstract}
Background: Metastatic renal cell carcinoma $(R C C)$ often develops resistance to first-line targeted therapy such as sunitinib. G-Protein-coupled estrogen receptor 1 (GPER1) agonist G-1 was recently reported to regulate $R C C$ physiology but the role of G-1 in RCC tumorigenesis and sunitinib resistance remains largely unknown. Materials and Methods: Parental and sunitinib-resistant 786-O cells were treated with GPERI agonist G-1, and quantitative phosphoproteomics was performed. Bioinformatic analyses and validations, including immunoblotting, cell migration, and cell cycle distribution, were performed. Results: $G-1$ repressed cell proliferation and migration in both parental and sunitinib-resistant 786-O cells. Phosphoproteomic signatures, including phosphoinositide 3-kinase and protein kinase B (PI3K-AKT) as well as other pathways, were upregulated in sunitinib-resistant cells but application of $G-1$ reversed this effect. Among phosphoprotein candidates, activating transcription factor 2 (ATF2) Thr69/71 phosphorylation was antagonistically regulated by sunitinib resistance and G-1. Conclusion: Our results open up the possibility for managing $R C C$ and sunitinib resistance by GPERI agonist $G-1$ and its regulated pathways.
\end{abstract}

This article is freely accessible online.

Correspondence to: Wei-Chi Ku, Ph.D., School of Medicine, College of Medicine, Fu Jen Catholic University, New Taipei City 242062, Taiwan. R.O.C. Tel: +886 229056451, Fax: +886 229052096, e-mail:089052@mail.fju.edu.tw

Key Words: G-Protein-coupled estrogen receptor 1, renal cell carcinoma, sunitinib resistance, quantitative phosphoproteomics.
Renal cell carcinoma (RCC) is the leading cause of kidney malignancy, contributing to an estimated $4-5 \%$ of new cases in 2020 (1). In addition to radical or partial nephrectomy, sunitinib is the first-line standard therapy for low-risk recurrent or metastatic RCC $(2,3)$. Primary or secondary resistance, however, usually develops after sunitinib administration (4). Although the combination of other target therapeutics, such as immune checkpoint inhibitors, seems to be a promising treatment option (5), there is still a need to investigate alternative strategies for managing RCC, especially in cases of sunitinib resistance.

The incidence of RCC has a male-to-female ratio of 2:1 in young patients but becomes approximately $1: 1$ in patients over 70 years old (6), implying the inhibitory role of estrogen in RCC carcinogenesis. Estrogen can repress RCC growth by non-genomic signaling pathways via classical, nuclear estrogen receptors (7-9). In addition to nuclear estrogen receptors, G-protein-coupled estrogen receptor 1 (GPER1) was identified as a non-classical estrogen receptor, and its expression correlated with tumorigenesis and poor prognosis in several cancer types, including breast and prostate cancer (10). Treatment of in vitro cancer cell lines by the GPER 1 agonist, G-1, led to variable results. For example, G-1 inhibited cell growth in prostate cancer (11) but promoted cell survival and migration in RCC cell lines (12). Recently, we demonstrated that estrogen inhibited RCC cell growth and alleviated sunitinib resistance in vitro through estrogen-triggered modulation of phosphorylation (9, 13). However, it remains unclear whether G-1 regulates phosphorylation dynamics in RCC tumorigenesis as well as and sunitinib resistance.

Quantitative phosphoproteomics has been used to study phosphorylation signaling in cancer development and drug resistance. Smith et al. found the novel phosphoproteomic 
signature in GPER1 agonist G-1-treated breast cancer cells (14). In addition, by combining immunoprecipitation with quantitative phosphoproteomics, a sunitinib-regulated tyrosine phosphorylation network was revealed (15). In the present study, we utilized a quantitative phosphoproteomics approach to survey G-1-regulated phosphorylation changes and their effect on sunitinib resistance with respect to cell proliferation, cell cycle, and cell migration. The identification of phosphoproteins that are regulated with G-1 may provide novel therapeutic targets for sunitinib-resistant RCC.

\section{Materials and Methods}

Chemicals. All chemicals, unless specified otherwise, were purchased from Sigma-Aldrich (St. Louis, MO, USA).

Generation of sunitinib-resistant RCC cells. The human RCC cell line 786-O was purchased from the Biological Collection and Research Center (Hsinchu, Taiwan) and maintained in RPMI 1640 medium (without phenol red) supplemented with $2 \mathrm{mM} \mathrm{L-glutamine}$ and $10 \%$ fetal bovine serum (Life Technologies, Grand Island, NY, USA). Sunitinib-resistant $786-O$ cells were generated in-house by gradually increasing exposure up to $10 \mu \mathrm{M}$ sunitinib in vitro (16). Sunitinib resistance (SunR) was confirmed by cell growth and sunitinib accumulation in lysosomes (17).

Treatment with $G-1$ and determination of cell viability. Both parental (PAR) and SunR 786-O cells were seeded at $4 \times 10^{3}$ cells/well in $100 \mu \mathrm{l}$ culture medium in 96-well plates and cultured for $24 \mathrm{~h}$. The cells were then treated with sunitinib $(2.5$ to $10 \mu \mathrm{M})$, estrogen ( 1 to $5 \mu \mathrm{M})$, GPER1 agonist G-1 ( 1 to $5 \mu \mathrm{M})$, or GPER1 antagonist G-15 (1 to $5 \mu \mathrm{M})$ for an additional $48 \mathrm{~h}$. Cell viability and number were measured by colorimetric 3-(4,5-dimethylthiazol2-yl)-2,5-diphenyltetrazolium) assay as previously described (18).

Peptide dimethyl labeling and phosphopeptide enrichment. For quantitative phosphoproteomic analysis, both PAR and SunR 786-O cells were treated either with vehicle only (i.e. $0.1 \%$ dimethyl sulfoxide) or with $2 \mu \mathrm{M} \mathrm{G}-1$ for $48 \mathrm{~h}$. Cellular proteins were then harvested in a lysis buffer containing $4 \%$ sodium dodecyl sulfate, 100 $\mathrm{mM}$ triethylammonium bicarbonate, as well as protease and phosphatase inhibitor cocktails, and sonicated using a Bioruptor Plus Sonicator (Diagenode, Denville, NJ, USA). After acetone precipitation, protein pellets were resuspended in $100 \mathrm{mM}$ triethylammonium bicarbonate, and $8 \mathrm{M}$ urea, followed by trypsin digestion and dimethyl labeling as previously described (9). Peptides from G-1-treated PAR 786-O cells, vehicle-treated SunR cells, and G-1-treated SunR cells were labeled with stale isotopic formaldehyde $\left({ }^{13} \mathrm{CD}_{2} \mathrm{O}\right.$, or heavy labeled). For the vehicle-treated PAR 786-O cells, the peptides were labeled with formaldehyde $\left(\mathrm{CH}_{2} \mathrm{O}\right.$, or light labeled). After dimethyl labeling, equal amounts $(500 \mu \mathrm{g})$ of the heavy- and light-labeled peptides were combined and subjected to sequential phosphopeptide enrichment by $\mathrm{Ga}$ - and $\mathrm{Fe}$-immobilized metal affinity chromatography as previously described (19). The eluted phosphopeptides were further fractionated into six fractions using the high-pH reverse-phase StageTip method (20).

Nanoscale liquid chromatography coupled to tandem mass spectrometry (nanoLC-MS/MS) and statistical analysis of phosphosites.
The high-pH reverse phase-fractionated phosphopeptides were analyzed using an LTQ-Orbitrap XL mass spectrometer (Thermo Fisher Scientific, Bremen, Germany) coupled on-line to a Dionex Ultimate 3000 RSLC nano system (Thermo Fisher Scientific). Phosphopeptides were separated by in-house-prepared capillary column $(100 \mu \mathrm{m} \times 15$ $\mathrm{cm}$ tip column) packed with 3- $\mu$ m ReproSil-Pur 120 C18-AQ reversephase beads (Dr. Maisch HPLC GmbH, Ammerbuch-Entringen, Germany), and data-dependent acquisition in LTQ-Orbitrap XL were performed as described previously (9). In the present study, three independent biological batches with duplicate nanoLC-MS/MS analyses were performed. Phosphopeptide identification and dimethyl quantification were performed using MaxQuant software (version 1.5.3.8) (21) against a reviewed SWISS-PROT sequence database (version 2016_05, with 20, 207 human canonical protein sequences) as previously described (9). The false discovery rate (FDR) at the peptide and protein levels was fixed at $1 \%$ in MaxQuant. The localization probability of each identified phosphorylation site (phosphosite) was also determined by MaxQuant. All nanoLC-MS/MS raw files as well as MaxQuant-generated result data were deposited onto the ProteomeXchange (22) Consortium via the Proteomics Identifications (PRIDE) partner repository with the dataset identifier PXD021254.

All phosphosite analyses were performed in Perseus platform (version 1.6.2.3) (23). Only high-confidence phosphosites (localization probability $>0.75$ or class I phosphosites) (24) were analyzed. For the SunR group, light-labeled intensity (PAR) and heavy-labeled intensity (SunR) were filtered to remove missing values. The phosphosite intensities were normalized by the internal reference scale (IRS) method (25), $\log 2$-transformed, and subjected to permutation FDR testing with s0 setting of 0.1 to identify dysregulated phosphosites $(p<0.01)$ (23). The G-1-treated group was analyzed using the same approach. For comparing the G-1treated and untreated SunR groups, differences in phosphosite levels were calculated as the 'ratio of ratios', according to a previous study (26). We first filtered for phosphosites with quantification information in the SunR (vs. PAR) and G-1-treated SunR (vs. PAR) groups. After IRS normalization across the two groups, the expression ratio for each group was calculated as SunR/PAR or (G1-treated SunR)/PAR. Finally, the obtained ratio of G-1-treated SunR was divided by that of SunR. The resulting expression ratios were subjected to permutation FDR testing.

Bioinformatics of differentially regulated phosphosites. Gene Ontology network analysis was performed on the dysregulated phosphosites using Cytoscape (v3 .8.0) (27) plug-in ClueGO (v2.5.7) $(28,29)$. Briefly, the regulated phosphosites were clustered (cut-off $p$-value $=0.01)$ using ontologies/pathways information from the Gene Ontology biological pathways, Kyoto Encyclopedia of Genes and Genomes (KEGG) (30, 31), Comprehensive Resource of Mammalian Protein Complexes (32), and WikiPathway (33) databases. Enriched clusters were annotated by AutoAnnotate (v1.3.3) (34), a Cytoscape application for summarizing networks with semantic annotations. In addition, the Database for Annotation, Visualization and Integrated Discovery (DAVID) functional annotation module (35) with default settings was used to analyze the Gene Ontology information of the regulated phosphosites. The kinase-substrate relationship of the regulated phosphosites were analyzed using the post-translational modification-signature enrichment analysis (PTM-SEA) algorithm (36) with default settings and a cut-off FDR of 0.01 . Finally, the network visualization of the regulated phosphosites was performed using the Cytoscape application PhosphoPath (v3.2) (37). 
Qualitative immunoblotting. Ten micrograms of cellular lysates were used for western blotting as previously described (9). The primary antibodies used in the present study and their dilution factors were as follows: anti-phospho (p)-activating transcription factor 2 (ATF2) (Thr69/71) (Cat. 9225) at 1:1000, anti-ATF2 (Cat. 9226) at 1:1500; anti-cyclin-dependent kinase 1 (CDK1) (Cat. 9116) at 1:1000 from Cell Signaling Technology (Danvers, MA, USA); anti-cyclin B (Cat. sc-245) at 1:1000 and anti-cyclin D (M20) (Cat. sc-718) from Santa Cruz Biotechnology (Dallas, TX, USA); and anti-glyceraldehyde 3-phosphate dehydrogenase (GAPDH) (Cat. 60004-1-Ig) at 1:40000 from Proteintech (Rosemont, IL, USA).

Cell migration assay. Migration of PAR and SunR 786-O cells following G-1 treatment was measured using the Oris ${ }^{\mathrm{TM}}$ Cell Migration Assay kit (Platypus Technologies, Madison, WI, USA) according to the manufacturer's protocol. Briefly, $5 \times 10^{4}$ cells were seeded onto each well of a 96-well plate and cultured for $24 \mathrm{~h}$. After the stopper was removed from each well, fresh culture media with or without $2 \mu \mathrm{M} \mathrm{G}-1$ was added to the cell culture for $48 \mathrm{~h}$. The cells were then stained with a cell-permeant derivate of calcein, calcein acetoxymethyl, and the number of migrated cells was determined by measuring fluorescence at $485 / 528 \mathrm{~nm}$ excitation/emission using an Infinite M200 PRO microplate reader (Tecan, Zürich, Switzerland).

Cell-cycle profiling by flow cytometry. Cell-cycle profiles were determined by propidium iodide (PI) DNA staining followed by flow cytometry (38). Briefly, $5 \times 10^{5}$ PAR or SunR 786-O cells were treated with or without $2 \mu \mathrm{M} \mathrm{G}-1$ for $48 \mathrm{~h}$. The cells were harvested by trypsinization and fixed with $70 \%$ ethanol. For PI staining, ethanolfixed cells were incubated in PI staining buffer containing $0.2 \mathrm{mg} / \mathrm{ml}$ RNase A, $0.1 \%$ Triton X-100, and $20 \mu \mathrm{g} / \mathrm{ml}$ PI in phosphate-buffered saline, for $30 \mathrm{~min}$ in the dark at room temperature. Fluorescence emitted at $585 \mathrm{~nm}$ from the PI-stained DNA was detected using a FACScan flow cytometer (BD Biosciences, San Jose, CA, USA). The percentages of cells (from a total of 104 cells) in the different cell phases were determined using FlowJo software, version 8.7 (BD Life Sciences, Ashland, OR, USA).

\section{Results}

GPER1 agonist G-1 inhibited cell growth of both PAR and SunR RCC cells. We first generated SunR 786-O cells by incubating the cells with half-maximal inhibitory concentration of sunitinib, i.e. $10 \mu \mathrm{M}$, for more than 6 months (16), and confirmed that SunR 786-O cells showed less growth inhibition by sunitinib than PAR cells $(p<0.01)$ (Figure 1A). In agreement with previous studies $(8,9,13)$, estrogen repressed growth of both PAR and SunR 786-O cells at relatively high concentrations (1 to $5 \mu \mathrm{M}$ ) (Figure $1 \mathrm{~B})$.

GPER1, a transmembrane estrogen receptor (39), was shown to participate in RCC cell physiology (12). In addition to endogenous estrogen, GPER1 can also be regulated by the synthetic agonist G-1 and antagonist G-15 (40, 41). We therefore investigated whether GPER1 agonist/antagonist is able to regulate growth of 786-O cells. The concentration ranges for GPER1 agonist/antagonist, i.e. 1 to $5 \mu \mathrm{M}$, were chosen based on previous studies $(12,14)$. The GPER1 agonist G-1 significantly inhibited cell growth of PAR as well as SunR cells, and SunR cells were more susceptible to $\mathrm{G}-1$, i.e. at $1 \mu \mathrm{M}$, than PAR cells (Figure 1C). Although GPER1 antagonist G-15 also caused growth inhibition in both PAR and SunR cells (Figure 1D), G-1 showed greater growth inhibition than G-15 at the same concentrations, especially in SunR cells (Figure 1C and 1D). Collectively, these data suggest that the GPER 1 agonist G-1 is a potential growth inhibitor in both PAR and SunR cells.

Quantitative phosphoproteomics revealed sunitinib resistance- and G-1-regulated phosphosites. Although G-1 is believed to be a specific GPER1 agonist (41), G-1 may affect both GPER1-dependent and -independent signaling pathways. To further dissect the possible phosphorylation signaling pathways regulated by G-1 treatment, chronic sunitinib treatment, and G-1 treatment in SunR cells, quantitative phosphoproteomics revealed 2893 and 3098 quantifiable phosphosites in the SunR and G-1 treatment groups, respectively. After IRS normalization (25), followed by permutation FDR testing $(p<0.01)$, we identified 615 upregulated and 886 down-regulated phosphosites in SunR 786-O cells (Figure 2A) as well as 629 up-regulated and 690 down-regulated phosphosites in G-1-treated PAR 786-O cells (vs. control) (Figure 2B).

We further analyzed the dysregulated phosphosites using ClueGO $(28,29)$ to explore the functions of phosphosites in SunR and G-1-treated PAR 786-O cells. For SunR 786-O cells, two clusters were up-regulated in response to chronic sunitinib treatment, including the adherens junction organization as well as vascular endothelial growth factor receptor (VEGFR) pathway and focal adhesion (Figure 2C). This agrees with previous observations, where sunitinibresistance up-regulated VEGF-related pathways $(4,17)$. In contrast, phosphosites involved in transcription factor binding and mRNA splicing were down-regulated, implying global repression of gene transcription in SunR cells (Figure 2A). Microtubule organization, negative regulation of organelle organization, as well as nuclear division and segregation were down-regulated in SunR cells (Figure 2A).

On the other hand, G-1 up-regulated phosphosites involved in protein sumoylation and ubiquitinylation, nucleocytoplasmic transport, DNA integrity checkpoints, and actin filament bundles (Figure 2B). Phosphosites involved in the receptorsignaling pathway, adhesion/tight junctions, and spliceosome were down-regulated in G-1-treated PAR cells (Figure 2B).

G-1 antagonized sunitinib resistance-related phosphorylation pathways. To explore the potential effect of G-1 on the sunitinib resistance-regulated phosphosites, the phosphosite intensities in G-1-treated SunR cells were compared to those of the untreated SunR cells. We identified 24 up-regulated $\left(\log _{2}\right.$ ratio $\left.>0.5\right)$ and 10 down-regulated $\left(\log _{2}\right.$ ratio $\left.<-0.5\right)$ 
A

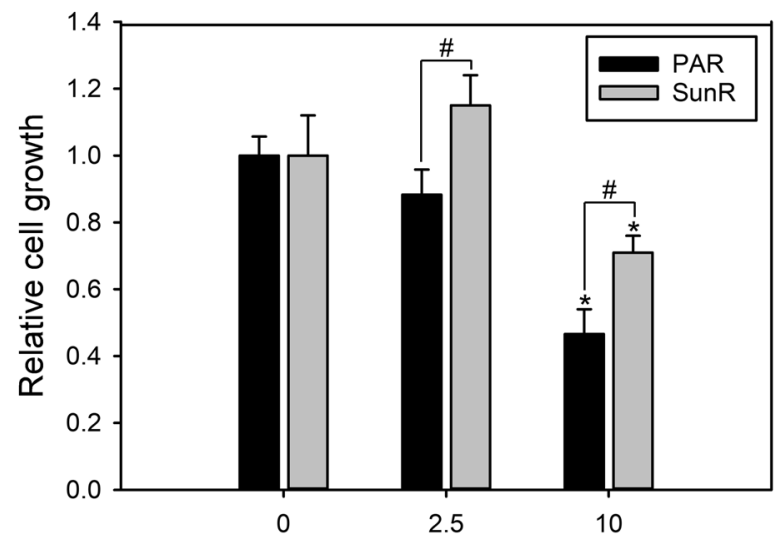

Sunitinib concentration $(\mu \mathrm{M})$

C

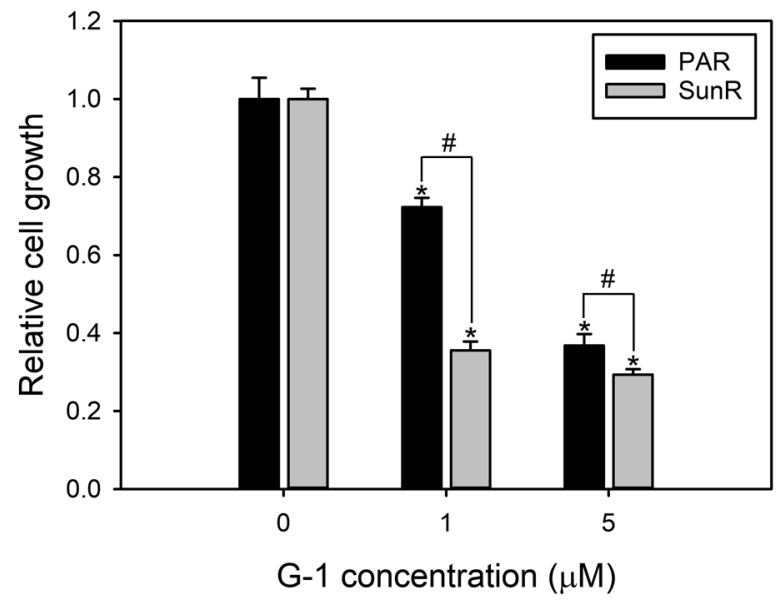

B

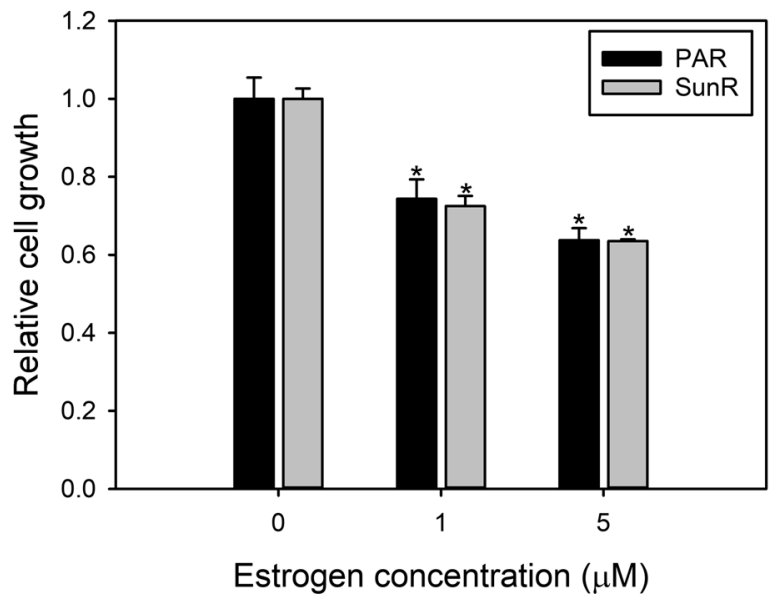

D

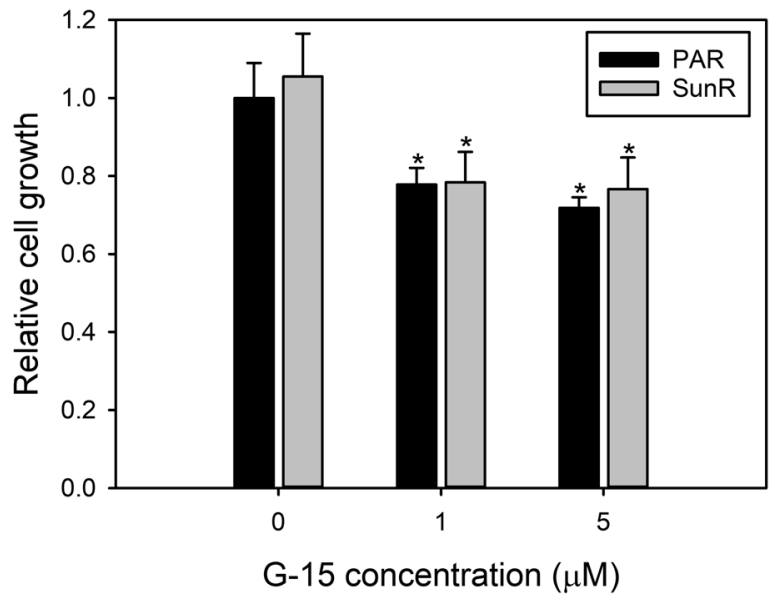

Figure 1. G-Protein-coupled estrogen receptor 1 (GPER1) agonist G-1 suppressed cell growth in both parental (PAR) and sunitinib-resistant (SunR) 786-O cells. SunR 786-O cells were first generated by chronic exposure to $10 \mu M$ sunitinib for over 6 months. Both PAR and SunR 786-O cells were incubated with sunitinib $(2.5$ or $10 \mu M)(A)$, estrogen $(1$ or $5 \mu M)(B)$, GPER1 agonist G-1 (1 or $5 \mu M)(C)$, or GPER1 antagonist G-15 (1 or $5 \mu M)(D)$ for 48 h, and then viable cells were determined by 3-(4,5-dimethylthiazol-2-yl)-2,5-diphenyltetrazolium bromide assay. All treatments were repeated in triplicate. Significantly different at $p<0.05$ from *vehicle control, and \#indicated group.

phosphosites $(p<0.01)$ (Figure 3A). Enrichment for protein functions and pathway information of the differentially expressed phosphosites was determined by Fisher's exact test $(p<0.01)$ using gene annotation databases (Table I). For example, arrest defective 1 and $N$-acetyltransferase human (ARD1-NATH) complex, Ku-antigen and $\mathrm{N}$-methyl-Daspartic acid receptor-regulated protein 1 (Ku-antigenNARG1) complex, as well as activating transcription factor 2, hexokinase-1 and voltage-dependent anion channel-1 (ATF2HK1-VDAC1) complex were enriched. In addition, mitogenactivated protein kinase (MAPK) signaling pathway and nucleotide-binding domain (NOD)-like receptor signaling pathway were enriched. Finally, proteins related to DNAdamage checkpoint and repair were enriched.

To address the potential kinase motif(s) for the regulated phosphosites, the differentially expressed phosphosites were subjected to PTM-SEA analysis (36). Kinase motifs related to the cyclin dependent kinase (CDK) family were downregulated in both SunR and PAR 786-O cells (Figure 3B). These data indicate that sunitinib resistance reduced cellcycle/division activity, and G-1 further reduced CDK activities, such as of CDK1, CDK2, CDK4, and CDK6. On the other hand, several kinases and pathways, including C-C chemokine receptor type 7 (CCR7), phosphoinositide 3-kinase 
A
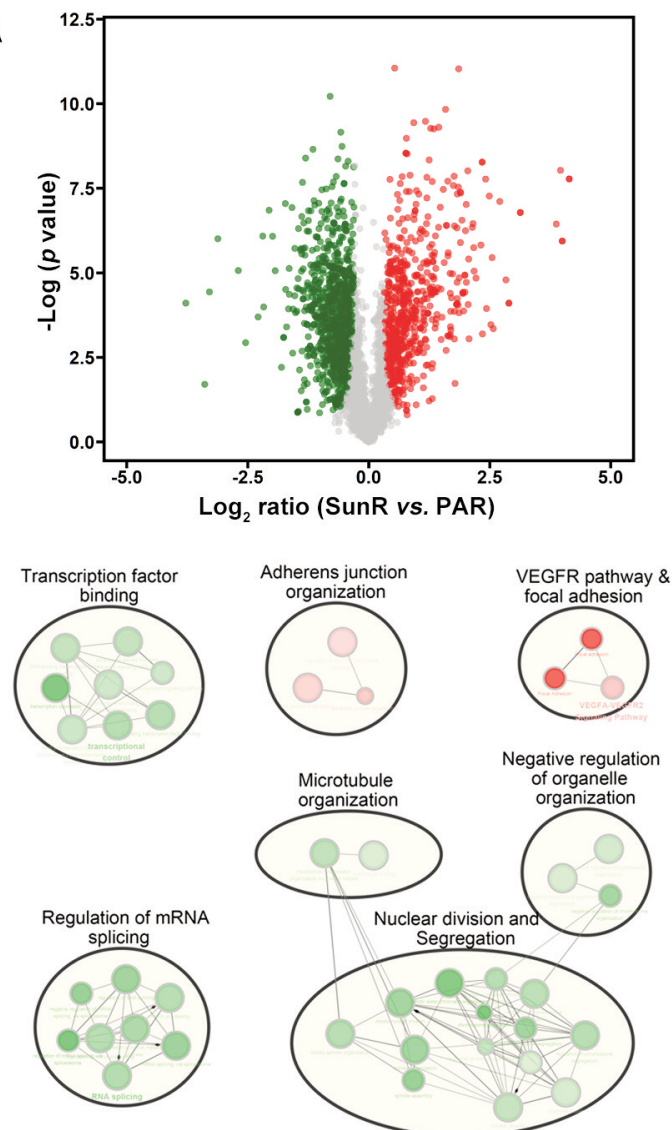

B
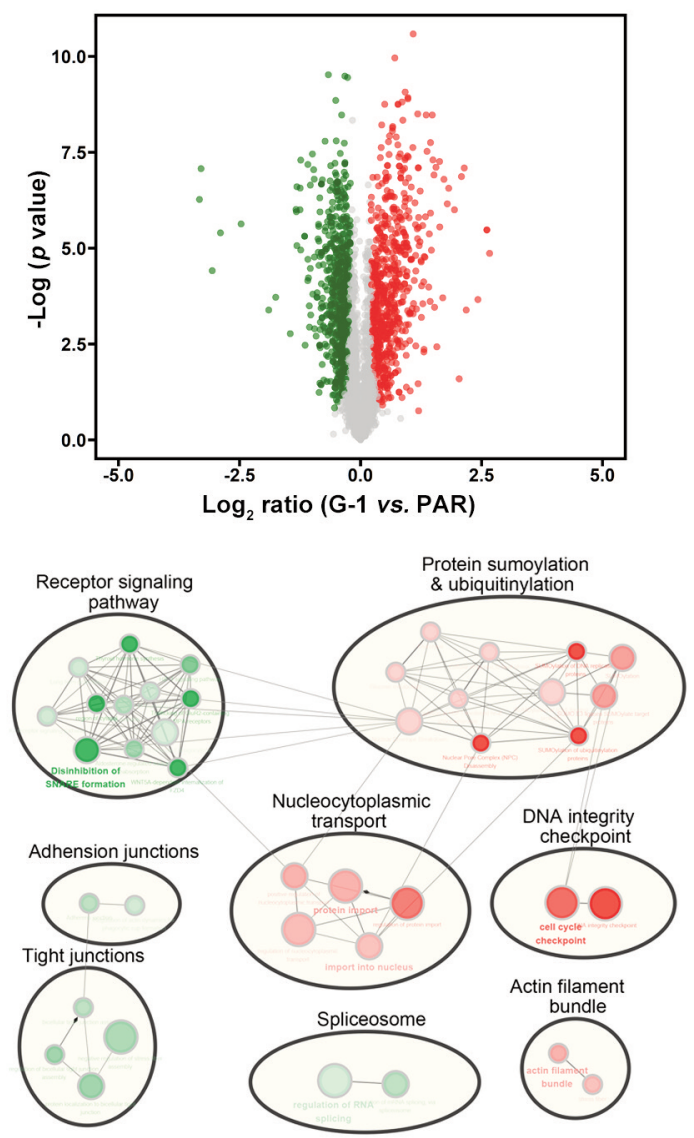

Figure 2. Quantitative phosphoproteomics revealed sunitinib resistance-and G-protein-coupled estrogen receptor 1 agonist G-1-regulated phosphosites in sunitinib-resistant (SunR) (A) and G-1-treated (B) 786-O cells compared with the parental cells (PAR). Upper panel: Volcano plots of differentially regulated phosphosites (false discovery rate $<0.01$ and $s 0=0.1$ ). Lower panel: The ClueGO enriched protein functions of the regulated phosphosites. Red and green colors denote up-regulated and down-regulated phosphosites, respectively.

and protein kinase B (PI3K-AKT), epidermal growth factor receptor (EGFR), tyrosine kinase with immunoglobulin-like and EGF-like domains 2 (TIE2), tyrosine-protein kinase KIT (KIT) receptor, and interleukin 33 (IL33) signaling pathways, as well as glycogen synthase kinase 3 beta (GSK3B) and MAPK1 kinases were notably up-regulated in SunR 786-O cells. EGFR and PIK3-AKT pathways are involved in sunitinib resistance $(42,43)$. CCR7, GSKB, and KIT pathways have been correlated with prognostic and therapeutic resistance in RCC cells (44-46). G-1 treatment also alleviated these sunitinib resistance-activated kinases/pathways. Finally, motifs related to mammalian target of rapamycin (mTOR) and polo-like kinase 1 (PLK1) were up-regulated in G-1-treated SunR 786-O cells compared to that in untreated cells, indicating that G-1 may activate the mTOR and PLK1 pathways in SunR 786-O cells.

We also performed hierarchical clustering of the G-1regulated phosphosites in SunR 786-O cells (Figure 3C). In general, SunR 786-O and G-1-treated SunR 786-O cells showed opposite expression profiles. For example, phosphosites in clusters 1 and 2 were up-regulated in SunR 786-O cells but down-regulated in G-1-treated SunR 786O cells. In clusters 3-5, phosphosite expression was downregulated in SunR 786-O but up-regulated in G-1-treated SunR 786-O cells (Figure 3C). These data suggest that G1 can antagonize changes in phosphorylation during chronic sunitinib treatment. In addition, PhosphoPath analysis (37) showed that 20 out of 34 regulated phosphosites showed functional connections centered on ATF2, filamin A (FLNA), p21 activated kinase 1 (PAK1), and SH3 domain-containing kinase-binding protein 1 (SH3KBP1) (Figure 3D). Finally, we used the DAVID functional annotation module with UniProtKB keywords (UP_KEYWORDS), KEGG pathway (KEGG_PATHWAY), and Gene Ontology (GOTERM_MF_DIRECT) to identify enrichment in phosphosite clusters (35). Functions in the MAPK signaling 
A

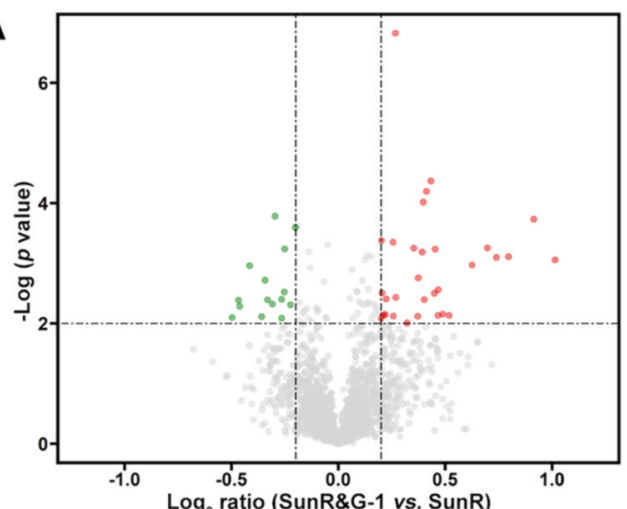

B

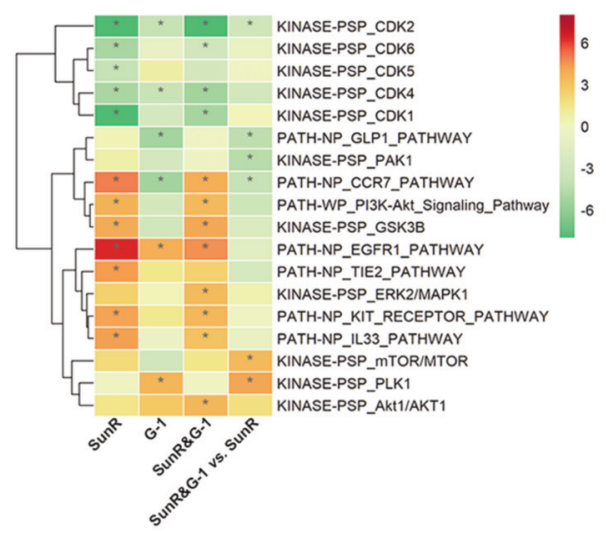

C

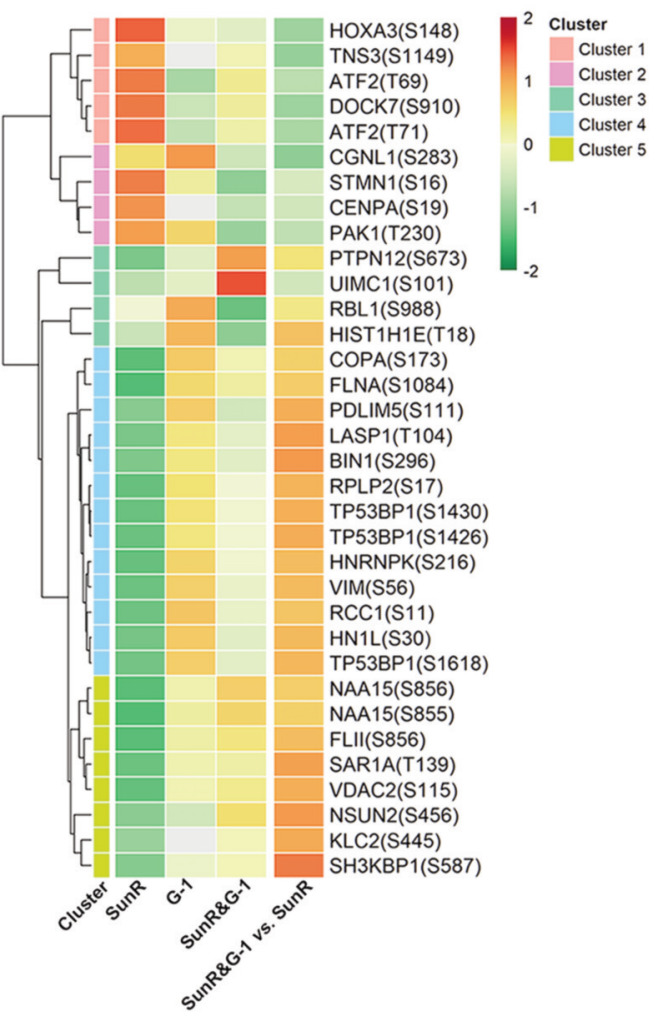

D

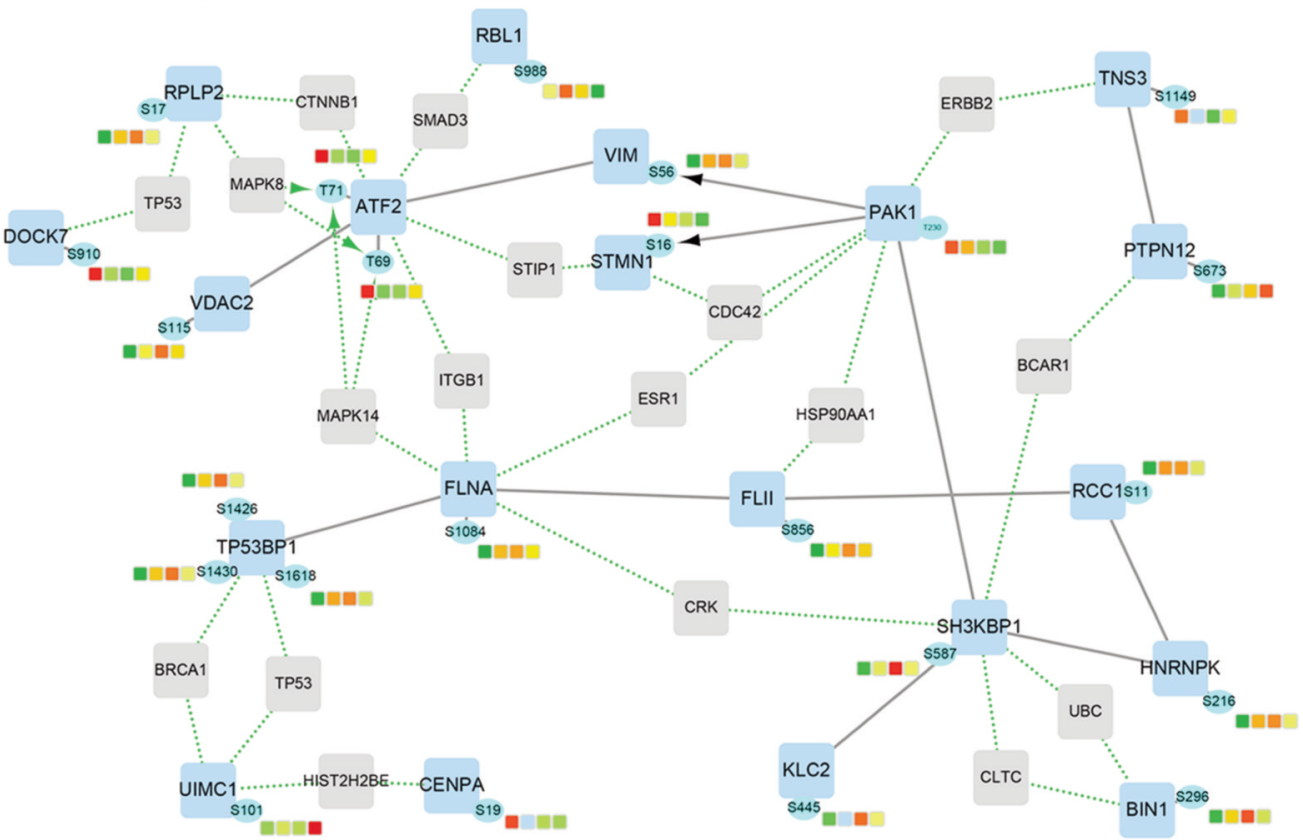

Figure 3. G-Protein-coupled estrogen receptor 1 agonist G-1-regulated phosphoproteomic signatures in sunitinib-resistant (SunR) 786-O cells. A: Volcano plot of $G$-1-regulated phosphosites ( $p<0.01$, Log2 ratio $>0.5$ or $<-0.5$ ) in G1-treated SunR 786-O cells compared with untreated SunR cells. B: Heatmap of phosphoproteomic signatures using the normalized enrichment scores by post-translational modification-signature enrichment analysis. The significantly enriched or depleted signatures at false discovery rate $<0.05$ are marked by asterisks. C: Hierarchical clustering of G1-regulated phosphosites in SunR cells. The comparative expression profiles of the selected phosphosites are also shown. D: The network of G-1regulated phosphosites in SunR cells depicted by PhosphoPath analysis. The relative expression of specific phosphosites is indicated by small colored boxes from left to right in: SunR, G-1-treated parental, G-1-treated SunR vs. untreated SunR (SunR\&G-1 vs. SunR), and G-1-treated SunR (SunR\&G1) 786-O cells, with red and green colors denoting up-regulation and down-regulation, respectively. 
Table I. Protein functions of phosphosites significantly regulated by G-protein-coupled estrogen receptor 1 agonist G-1 in sunitinib-resistant 786$O$ cells.

\begin{tabular}{lc}
\hline Source & Enriched protein functions \\
\hline CORUM & ARD1-NATH complex \\
CORUM & Ku antigen-NARG1 complex \\
CORUM & ATF2-HK1-VDAC1 complex \\
CORUM & TP53BP1-containing complex \\
CORUM & PTIP-DNA damage-response complex \\
CORUM & MDC1-TP53BP1-SMC1 complex \\
CORUM & MDC1-H2AX-TP53BP1 complex \\
CORUM & Prohibitin 2 complex, mitochondrial \\
KEGG brite & MAPK signaling pathway \\
KEGG brite & NOD-like receptor signaling pathway \\
KEGG brite & Cortisol synthesis and secretion \\
KEGG brite & Insulin secretion \\
KEGG brite & Cocaine addiction \\
KEGG brite & Viral carcinogenesis \\
KEGG brite & Aldosterone synthesis and secretion \\
KEGG brite & Thyroid hormone synthesis \\
KEGG brite & Amphetamine addiction \\
Reactome & G $/$ M DNA damage checkpoint \\
Reactome & Nonhomologous end-joining \\
Reactome & SUMOylation of transcription factors \\
Reactome & Processing of ends of DNA double-strand breaks \\
Reactome & Activation of the AP-1 family of transcription factors \\
Reactome & Recruitment and ATM-mediated phosphorylation of repair and signaling proteins at DNA double strand breaks \\
\hline
\end{tabular}

AP-1: Activator protein 1; ARD1: arrest defective 1; ATF2: activating transcription factor 2; ATM: ataxia telangiectasia mutated; CORUM: Comprehensive Resource of Mammalian Protein Complexes; H2AX: histone H2AX; HK1: hexokinase-1; KEGG: Kyoto Encyclopedia of Genes and Genomes; MAPK: mitogen-activated protein kinase; MDC1: mediator of DNA damage-checkpoint protein 1; NARG1: $N$-methyl-D-aspartic acid receptor-regulated protein 1; NATH: $N$-acetyltransferase human; NOD: nucleotide-binding domain; PTIP: PAX2 transactivation domain-interacting protein; SMC1: structural maintenance of chromosomes protein 1; TP53BP1: p53-binding protein 1; VDAC1: voltage-dependent anion channel-1.

pathway, acetylation, DNA-binding, and alternative splicing, were enriched in clusters 1 and 2 (Table II). On the other hand, functions in the SH3 domain, cell-cell adhesion, transcription regulation, and methylation were enriched in clusters 3-5.

Integral network analysis highlighted the crosstalk of phosphorylation dynamics between G-1-treated PAR 786$O$ cells, SunR 786-O cells, and G-1-treated SunR 786-O cells. We dissected the possible crosstalk between G-1 and sunitinib in RCC cells by performing integral network analysis. We used the phosphosites with significant changes in at least one of the following conditions: G-1treated PAR vs. PAR 786-O cells, SunR 786-O vs. PAR 786-O, and G-1-treated SunR vs. untreated SunR 786-O cells. A total of 2,286 phosphosites were analyzed by the PhosphoPath analysis. In agreement with PTM-SEA analysis (Figure 3B), PhosphoPath analysis indicated enrichment of the VEGFA-VEGFR2 signaling pathway $(p<0.0001)$, focal adhesion-PIK3-AKT-mTOR signaling pathway $(p<0.0001)$, and cell cycle checkpoint $(p<0.0001)$ (Figure 4).
G-1 inhibited ATF2 phosphorylation and cell-cycle progression. According to our phosphoproteomics data, ATF2 phosphorylation at Thr69 and Thr71 was differentially regulated in G-1-treated and untreated SunR 786-O cells (Figure 3C and 3D). In addition, ATF2 was also found in the VEGFA-VEGFR2 signaling pathway (Figure 4A) and focal adhesion-PIK3-AKT-mTOR signaling pathway (Figure 4B), suggesting that ATF2 may be affected by G-1-mediated and sunitinib resistance-related signaling pathways. In agreement with the phosphoproteomics data, we confirmed that Thr69/71 phosphorylation in ATF2 was up-regulated in SunR 786-O cells, and that G-1 treatment inhibited Thr69/71 phosphorylation in both PAR and SunR 786-O cells (Figure 5A). In contrast, ATF2 expression did not significantly change by treatment, demonstrating that G-1 and sunitinib resistance affect ATF2 activity by protein phosphorylation.

Recently, Hasegawa et al. reported that CDK1 and cyclin B complex promote cell-cycle progression into $\mathrm{M}$ phase by phosphorylating Thr69/71 in ATF2 (47). In addition, our phosphoproteomics data suggested that G-1 regulates the 
Table II. Functional annotation of G-1-regulated phosphosite clusters in sunitinib-resistant 786-O cells by the Database for Annotation, Visualization and Integrated Discovery.

\begin{tabular}{|c|c|c|c|c|}
\hline Category & Term & Genes & $\begin{array}{l}\text { Fold } \\
\text { enrichment }\end{array}$ & $\begin{array}{l}\text { Enrichment } \\
\text { score }\end{array}$ \\
\hline \multicolumn{5}{|l|}{ Cluster 1-2 } \\
\hline KEGG_PATHWAY & MAPK signaling pathway & PAK1, STMN1, ATF2 & 17.2 & 0.74 \\
\hline UP_KEYWORDS & Acetylation & PAK1, STMN1, DOCK7, ATF2 & 1.2 & 0.74 \\
\hline UP_KEYWORDS & DNA-binding & CENPA, ATF2, НОХАЗ & 3.6 & 0.27 \\
\hline UP_KEYWORDS & Alternative splicing & $\begin{array}{l}\text { PAK1, STMN1, TNS3, } \\
\text { DOCK7, CENPA, ATF2 }\end{array}$ & 1.2 & 0.20 \\
\hline \multicolumn{5}{|l|}{ Cluster 3-5 } \\
\hline UP_KEYWORDS & SH3 domain & SH3K1, LASP1, BIN1 & 4.1 & 0.83 \\
\hline GOTERM_MF_DIRECT & $\begin{array}{l}\text { Cadherin binding involved } \\
\text { in cell-cell adhesion }\end{array}$ & $\begin{array}{l}\text { KLC2, PDLI5, FLNA, } \\
\text { LASP1, HNRNPK }\end{array}$ & 2.2 & 0.69 \\
\hline UP_KEYWORDS & Transcription regulation & $\begin{array}{l}\text { FLII, TP53BP1, NAA15, } \\
\text { RBL1, UIMC1, HNRNPK }\end{array}$ & 1.5 & 0.31 \\
\hline UP_KEYWORDS & Methylation & $\begin{array}{l}\text { HIST1H1E, COPA, TP53BP1, } \\
\text { RCC1, LASP1, HNRNPK }\end{array}$ & 1.5 & 0.30 \\
\hline
\end{tabular}

ATF2: Activating transcription factor 2; BIN1: myc box-dependent-interacting protein 1; CENPA: centromere protein A; COPA: coatomer subunit alpha; DOCK7: dedicator of cytokinesis 7; FLII: Protein flightless-1 homolog; FLNA: filamin A; HIST1H1E: histone H1.4; HNRNPK: heterogeneous nuclear ribonucleoprotein K; HOXA3: homeobox A3; KLC2: kinesin light chain 2; LASP1: LIM and SH3 domain protein 1; MAPK: mitogen-activated protein kinase; NAA15: N-alpha-acetyltransferase 15; PAK1: p21 activated kinase 1; PDLI5: PDZ and LIM domain protein 5; RBL1: retinoblastomalike protein 1; RCC1: regulator of chromosome condensation ; SH3K1: SH3 domain-containing kinase-binding protein 1; STMN1: stathmin 1; TNS1: tensin 1; UIMC1: ubiquitin interaction motif containing 1 .

cell-cycle checkpoint (Figure 4C). Therefore, we performed flow cytometry to examine the cell-cycle progression in cells. Firstly, G-1 treatment promoted S-phase entry in PAR 786-O cells (Figure 5B). The entry of S-phase after G-1 treatment may be explained by the increased expression of G1/S-phase cyclin D1 in response to G-1 treatment in PAR 786-O cells (Figure 5A). On the other hand, G-1 treatment blocked the $\mathrm{G} 2 / \mathrm{M}$ phase transition in PAR 786-O cells (Figure 5B). This G2/M arrest coincided with reduced CDK1 and cyclin B expression by G-1 treatment, as well as the down-regulation of ATF2 Thr69/71 phosphorylation (Figure $5 \mathrm{~A})$. In contrast, G-1 treatment did not lead to any significant changes in cell-cycle progression in SunR 786-O cells (Figure 5B), although ATF2 phosphorylation as well as cellcycle regulators, such as CDK1, cyclin B and cyclin D were inhibited by G-1 treatment in SunR cells.

Effect of G-1 on RCC cell migration. According to our phosphoproteomics data, both G-1 treatment and sunitinib resistance may affect cell migration and cell adhesion. We therefore monitored cell migration using Oris ${ }^{\mathrm{TM}}$ cell migration assays (48). Sunitinib resistance reduced cell migration by $\sim 50 \%$ in $786-\mathrm{O}$ cells, and G-1 inhibited cell migration by nearly $50 \%$ in both PAR and SunR 786-O cells (Figure 5C and D). Taken together, these data show G-1 can repress cell migration in both PAR and SunR 786-O cells.

\section{Discussion}

It has been shown that estrogen represses RCC cell growth via classical estrogen receptors $(8,9)$, and perturbs sunitinib resistance in in vitro RCC cell culture (13). The role of nonclassical estrogen receptor GPER1 in RCC physiology, however, is still unclear. In the present study, we showed that GPER 1 agonist G-1 repressed cell growth in both PAR and SunR 786-O RCC cells. Using quantitative phosphoproteomics, we further demonstrated that G-1 perturbs phosphoproteomic signatures involved in VEGFR pathway, cell-cycle progression, as well cell migration. Although the exact role of GPER1 remains to be further addressed, our observations imply the importance of the GPER1 pathway in regulating $\mathrm{RCC}$ growth. Because estrogen may induce genomic changes and cause delirious side-effects via classical estrogen receptors (49), we anticipate that the G-1-regulated extranuclear-initiated ('nongenomic') pathway, either GPER1dependent or -independent, provides an alternative approach for managing RCC.

Our phosphoproteomics data also highlighted the potential roles of ATF2 in sunitinib resistance and G-1-regulated signaling pathways. Elevated ATF2 expression contributes to resistance to sorafenib, another first-line tyrosine kinase inhibitor, in liver cancer (50). Silencing of ATF2 inhibited the growth of pancreatic cancer cells and enhanced 

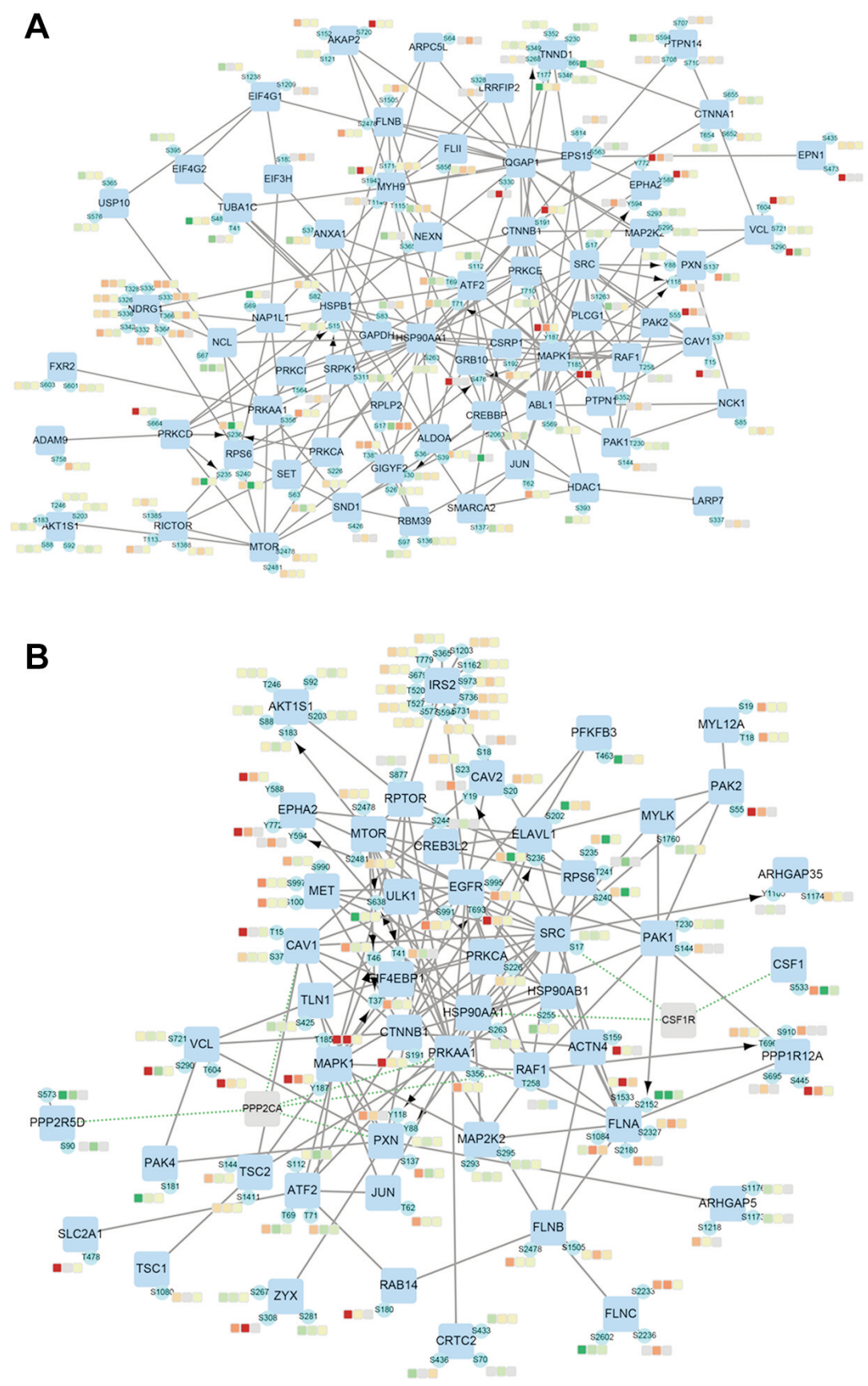

Figure 4. Continued

sensitivity to chemotherapy (51). In addition, up-regulation of ATF2 expression is also correlated with poor prognosis of RCC (52). Together, these observations demonstrated that ATF2 might participate in RCC carcinogenesis and tyrosine kinase inhibitor resistance, although regulation of ATF2 phosphorylation adds another level of regulation to this system. In the present study, we observed the up-regulation of Thr69/71 phosphorylation of ATF2 in sunitinib resistance, and treatment with G-1 reversed this. ATF2 Thr69/71 phosphorylation is required for its association with activator protein 1 (AP-1), nuclear localization, and transcriptional activation of downstream genes related to DNA repair and apoptotic signaling (53), which may promote resistance to therapeutic agents. The down-regulation of Thr69/71 


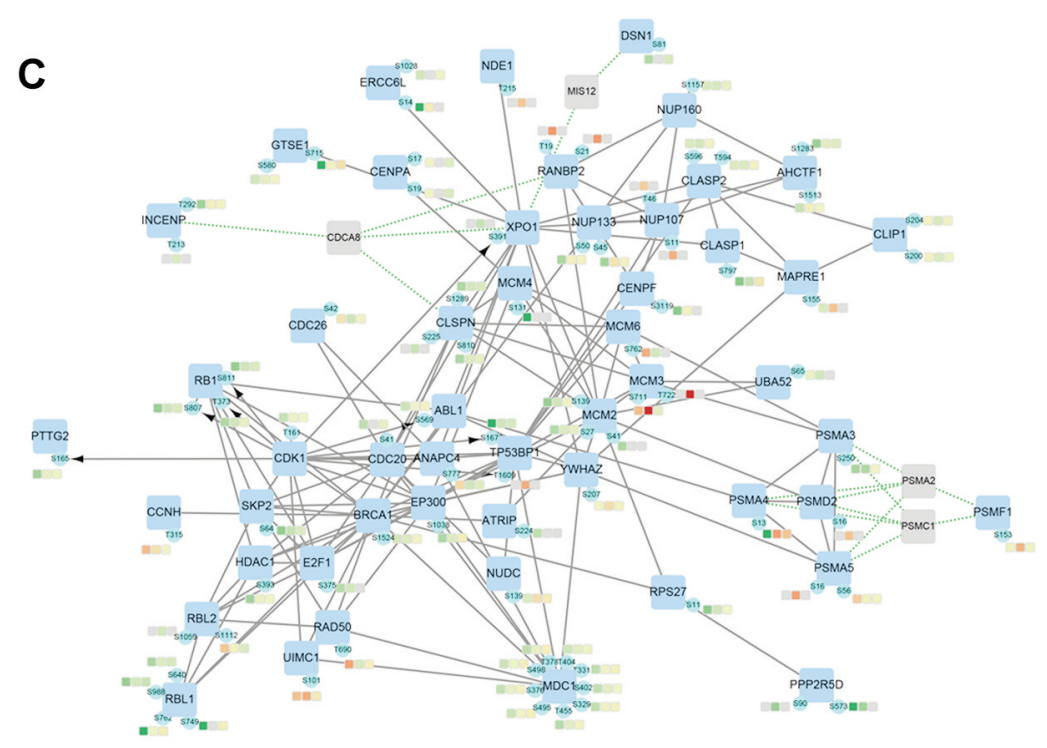

Figure 4. G-Protein-coupled estrogen receptor 1 agonist G-1 perturbed phosphorylation networks. Significantly altered selected phosphosites were analyzed by PhosphoPath. Enriched phosphorylation networks from vascular endothelial growth factor A-vascular endothelial growth factor receptor 2 (VEGFA-VEGFR2) pathway (A), focal adhesion-phosphoinositide 3-kinase-protein kinase B-mammalian target of rapamycin (PIK3-AKT-mTOR) signaling pathway $(B)$, and cell-cycle checkpoint $(C)$ are shown. The relative expression of each phosphosite is indicated by small colored boxes from left to right in: Sunitinib-resistant (SunR), G-1-treated parental, G-1-treated SunR vs. untreated SunR, and G-1-treated SunR 786-O cells, with red and green colors denoting up-regulation and down-regulation, respectively.

phosphorylation, as shown by G-1 treatment, may restrict ATF2 to the cytoplasm, and possibly be correlated with mitochondrial death (54). According to our results, ATF2 phosphorylation participated in the focal adhesion pathway, which affects cell migration. Therefore, the exact role of ATF2 phosphorylation in the GPER1 pathway warrants future studies.

Sunitinib resistance is correlated with cell survival and migration. However, there is controversy over how sunitinib resistance contributes to cell migration; in some studies, sunitinib resistance increased the cell migration rate $(55,56)$, while others have reported its reduction (57). In the present study, we demonstrated that sunitinib resistance in 786-O cells reduced cell migration. These results may be dependent on the sunitinib concentration, as low-dose sunitinib $(1 \mu \mathrm{M})$ seemed to promote cell migration, while high concentrations, such as $10 \mu \mathrm{M}$, inhibited cell migration. As the half-maximal inhibitory concentration of sunitinib is $\sim 10 \mu \mathrm{M}$ in the 786$\mathrm{O}$ cell lines (Figure 1A), we anticipate that high-dose sunitinib would provide a better model mimicking acquired resistance, as shown in previous studies (16).

Although G-1 inhibits cell growth and antagonizes sunitinib-resistant pathways, our phosphoproteomics analysis demonstrated that mTOR kinase was up-regulated in SunR 786-O cells, but inhibited in G-1-treated SunR 786-O cells. When compared to SunR cells, however, G-1 treatment of
SunR cells further up-regulated mTOR kinase activity. Similar results were also observed for PLK activity. RCC is known to activate the mTOR pathway during the development of resistance to therapies (4). In a recent study, activation of the PLK pathway was shown to promote proliferation and suppress apoptosis in RCC (58). Taken together, the significant up-regulation of mTOR kinase and PLK kinase in G-1-treated SunR 786-O cells raises concerns about the potential side-effects of G-1 on sunitinib-resistant cells.

Some limitations are present in this study. Although G-1 has been recognized as a specific GPER1 agonist, it may regulate RCC growth and phosphorylation dynamics in a GPER1-independent manner. Therefore, further studies are required to dissect the exact role of G-1 by using RCC cells with different GPER1 expression levels, such as GPERI knock-down or overexpression.

\section{Conclusion}

In conclusion, we demonstrated that the GPER 1 agonist G1 can regulate phosphorylation pathways related to RCC cell growth and migration, and antagonize the phosphorylation pathways activated by sunitinib resistance. Our results suggest a promising strategy for managing RCC and sunitinib resistance by GPER 1 agonist G-1 and its regulated pathways. 
A

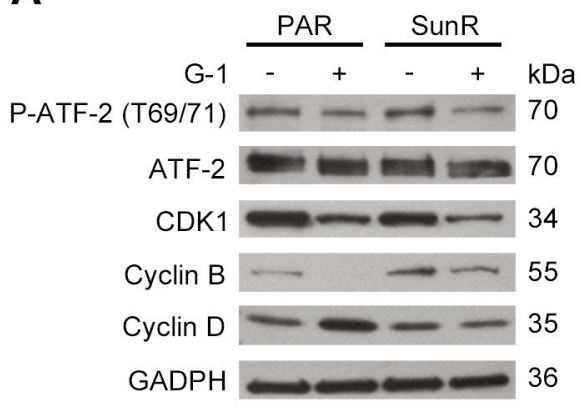

C

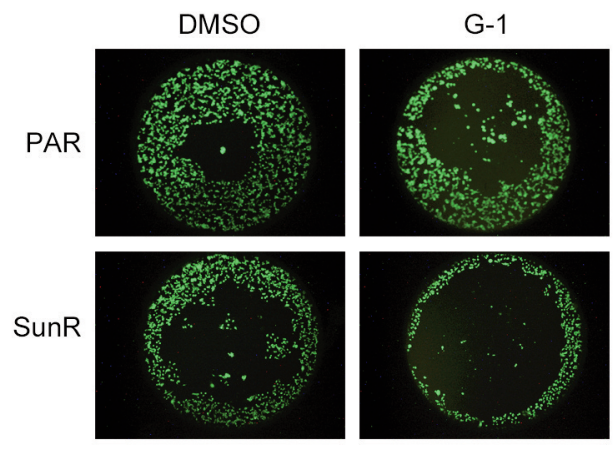

B

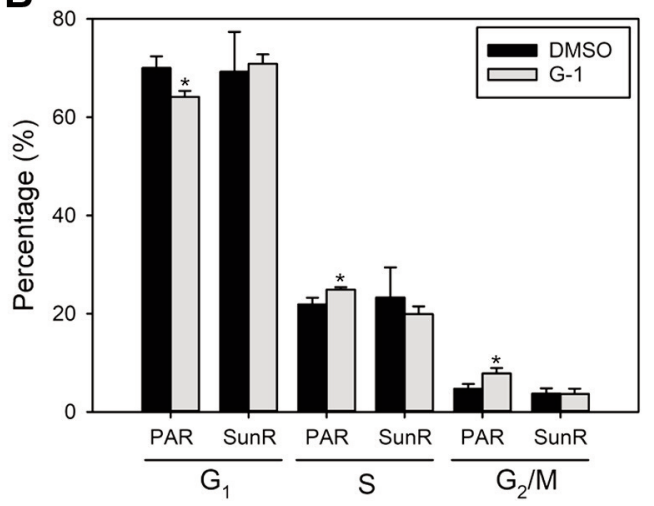

D

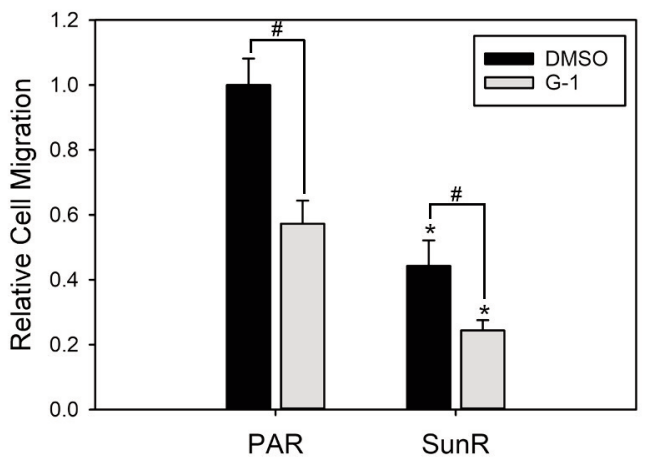

Figure 5. G-Protein-coupled estrogen receptor 1 agonist G-1 down-regulated cell-cycle progression and cell migration. A: Parental (PAR) and sunitinib-resistant (SunR) 786-O cells were treated with dimethyl sulfoxide (DMSO) or $2 \mu M$ G-1 for 48 h, and subjected to the designated analyses. A: Immunoblotting of cell-cycle-related proteins phospho-activating transcription factor 2 (P-ATF2) at Thr69 and 71 (T69/71), ATF2, cyclin-dependent kinase 1 (CDK1), cyclin B, cyclin D, and glyceraldehyde 3-phosphate dehydrogenase (GAPDH). B: Quantitation of cells in each cell-cycle phase (average $\pm S D$ ) from flow cytometry. C: Typical photograph of migrated cells stained by calcein-AM. D: Quantification (average $\pm S D$ ) of migrated cells shown in $(C)$. All treatments were repeated in triplicate. Significantly different at $p<0.05$ from *vehicle control, and \#indicated group.

\section{Conflicts of Interest}

The Authors declare that they have no competing interests in regard to this study.

\section{Authors' Contributions}

SKC, YCW, and WCK designed the experiments. TYL and HJW performed experiments. CJH, and WCK analyzed the data. SKC, $\mathrm{CJH}$, and WCK wrote the article.

\section{Acknowledgements}

The Authors thank the Mass Spectrometry Laboratory of Tzong Jwo Jang, College of Medicine, Fu Jen Catholic University, for the instrumental assistance for nanoLC-MS/MS analyses. This work was supported by the Ministry of Science and Technology, Taiwan (MOST105-2314-B-030-011 and MOST106-2314-B-030-009MY3); Cathay General Hospital, Taiwan (103-CGH-FJU-15 and
104-CGH-FJU-07); and Fu Jen Catholic University, New Taipei, Taiwan (A0109005).

\section{References}

1 Siegel RL, Miller KD and Jemal A: Cancer statistics, 2020. CA Cancer J Clin 70(1): 7-30, 2020. PMID: 31912902. DOI: $10.3322 / \mathrm{caac} .21590$

2 Vuorinen RL, Paunu N, Turpeenniemi-Hujanen T, Reunamo T, Jekunen A, Kataja V, Sintonen H, Purmonen T and KellokumpuLehtinen PL: Sunitinib first-line treatment in metastatic renal cell carcinoma: Costs and effects. Anticancer Res 39(10): 55595564, 2019. PMID: 31570450. DOI: 10.21873/anticanres.13749

3 Escudier B, Porta C, Schmidinger M, Rioux-Leclercq N, Bex A, Khoo V, Grünwald V, Gillessen S, Horwich A and ESMO Guidelines Committee: Renal cell carcinoma: ESMO clinical practice guidelines for diagnosis, treatment and follow-up. Ann Oncol 30(5): 706-720, 2019. PMID: 30788497. DOI: 10.1093/annonc/mdz056 
4 Makhov P, Joshi S, Ghatalia P, Kutikov A, Uzzo RG and Kolenko VM: Resistance to systemic therapies in clear cell renal cell carcinoma: Mechanisms and management strategies. Mol Cancer Ther 17(7): 1355-1364, 2018. PMID: 29967214. DOI: 10.1158/1535-7163.MCT-17-1299

5 Motzer RJ, Tannir NM, McDermott DF, Arén Frontera O, Melichar B, Choueiri TK, Plimack ER, Barthélémy P, Porta C, George S, Powles T, Donskov F, Neiman V, Kollmannsberger CK, Salman P, Gurney H, Hawkins R, Ravaud A, Grimm MO, Bracarda S, Barrios CH, Tomita Y, Castellano D, Rini BI, Chen AC, Mekan S, McHenry MB, Wind-Rotolo M, Doan J, Sharma P, Hammers HJ, Escudier B and CheckMate 214 Investigators: Nivolumab plus ipilimumab versus sunitinib in advanced renalcell carcinoma. N Engl J Med 378(14): 1277-1290, 2018. PMID: 29562145. DOI: 10.1056/NEJMoa1712126

6 Hew MN, Zonneveld R, Kümmerlin IP, Opondo D, de la Rosette $\mathrm{JJ}$ and Laguna MP: Age and gender related differences in renal cell carcinoma in a European cohort. J Urol 188(1): 33-38, 2012. PMID: 22578731. DOI: 10.1016/j.juro.2012.02.2573

7 Yu CP, Ho JY, Huang YT, Cha TL, Sun GH, Yu DS, Chang FW, Chen SP and Hsu RJ: Estrogen inhibits renal cell carcinoma cell progression through estrogen receptor- $\beta$ activation. PLoS One 8(2): e56667, 2013. PMID: 23460808. DOI: 10.1371/ journal.pone.0056667

8 Wu ST, Ku WC, Huang CJ, Wang YC, Lin CM and Chen SK: Cellular effects induced by $17-\beta$-estradiol to reduce the survival of renal cell carcinoma cells. J Biomed Sci 23(1): 67, 2016. PMID: 27680214. DOI: 10.1186/s12929-016-0282-z

9 Chen KC, Lin CM, Huang CJ, Chen SK, Wu ST, Chiang HS and $\mathrm{Ku}$ WC: Dual roles of $17-\beta$ estradiol in estrogen receptordependent growth inhibition in renal cell carcinoma. Cancer Genomics Proteomics 13(3): 219-230, 2016. PMID: 27107064.

10 Prossnitz ER: GPER modulators: Opportunity Nox on the heels of a class Akt. J Steroid Biochem Mol Biol 176: 73-81, 2018. PMID: 28285016. DOI: 10.1016/j.jsbmb.2017.03.005

11 Chan QK, Lam HM, Ng CF, Lee AY, Chan ES, Ng HK, Ho SM and Lau KM: Activation of GPR30 inhibits the growth of prostate cancer cells through sustained activation of Erk1/2, cjun/c-fos-dependent upregulation of p21, and induction of $\mathrm{G}(2)$ cell-cycle arrest. Cell Death Differ 17(9): 1511-1523, 2010. PMID: 20203690. DOI: $10.1038 /$ cdd 2010.20

12 Guan BZ, Yan RL, Huang JW, Li FL, Zhong YX, Chen Y, Liu FN, Hu B, Huang SB and Yin LH: Activation of G protein coupled estrogen receptor (GPER) promotes the migration of renal cell carcinoma via the PI3K/AKT/MMP-9 signals. Cell Adh Migr 12(2): 109-117, 2018. PMID: 25588050. DOI: $10.4161 / 19336918.2014 .990781$

$13 \mathrm{Ku}$ WC, Chen SK, Lin CM, Tang C and Wang YC: Label-free quantitative phosphoproteomics reveals the role of beta-estradiol in sunitinib-resistant renal cell carcinoma growth via perturbing transforming growing factor-beta pathway. Urol Sci 30(3): 107113,2019

14 Smith LC, Ralston-Hooper KJ, Ferguson PL and Sabo-Attwood $\mathrm{T}$ : The $\mathrm{G}$ protein-coupled estrogen receptor agonist G-1 inhibits nuclear estrogen receptor activity and stimulates novel phosphoproteomic signatures. Toxicol Sci 151(2): 434-446, 2016. PMID: 27026707. DOI: 10.1093/toxsci/kfw057

15 van der Mijn JC, Broxterman HJ, Knol JC, Piersma SR, De Haas RR, Dekker H, Pham TV, Van Beusechem VW, Halmos B, Mier JW, Jiménez CR and Verheul HM: Sunitinib activates Axl signaling in renal cell cancer. Int J Cancer 138(12): 3002-3010, 2016. PMID: 26815723. DOI: 10.1002/ijc.30022

16 Sakai I, Miyake $\mathrm{H}$ and Fujisawa M: Acquired resistance to sunitinib in human renal cell carcinoma cells is mediated by constitutive activation of signal transduction pathways associated with tumour cell proliferation. BJU Int 112(2): E211-E220, 2013. PMID: 23305097. DOI: 10.1111/j.1464-410X.2012.11655.x

17 Joosten SC, Hamming L, Soetekouw PM, Aarts MJ, Veeck J, van Engeland $\mathrm{M}$ and Tjan-Heijnen VC: Resistance to sunitinib in renal cell carcinoma: From molecular mechanisms to predictive markers and future perspectives. Biochim Biophys Acta 1855(1): 1-16, 2015. PMID: 25446042. DOI: 10.1016/j.bbcan.2014.11.002

18 Niks $\mathrm{M}$ and Otto M: Towards an optimized MTT assay. J Immunol Methods 130(1): 149-151, 1990. PMID: 2358686. DOI: $10.1016 / 0022-1759(90) 90309-j$

19 Tsai CF, Hsu CC, Hung JN, Wang YT, Choong WK, Zeng MY, Lin PY, Hong RW, Sung TY and Chen YJ: Sequential phosphoproteomic enrichment through complementary metaldirected immobilized metal ion affinity chromatography. Anal Chem 86(1): 685-693, 2014. PMID: 24313913. DOI: 10.1021/ ac4031175

20 Dimayacyac-Esleta BR, Tsai CF, Kitata RB, Lin PY, Choong WK, Lin TD, Wang YT, Weng SH, Yang PC, Arco SD, Sung TY and Chen YJ: Rapid high-pH reverse phase stagetip for sensitive small-scale membrane proteomic profiling. Anal Chem 87(24): 12016-12023, 2015. PMID: 26554430. DOI: 10.1021/acs.anal chem.5b03639

21 Cox J and Mann M: MaxQuant enables high peptide identification rates, individualized p.p.b.-range mass accuracies and proteomewide protein quantification. Nat Biotechnol 26(12): 1367-1372, 2008. PMID: 19029910 . DOI: $10.1038 /$ nbt.1511

22 Deutsch EW, Csordas A, Sun Z, Jarnuczak A, Perez-Riverol Y, Ternent T, Campbell DS, Bernal-Llinares M, Okuda S, Kawano S, Moritz RL, Carver JJ, Wang M, Ishihama Y, Bandeira N, Hermjakob H and Vizcaíno JA: The proteomeXchange consortium in 2017: Supporting the cultural change in proteomics public data deposition. Nucleic Acids Res 45(D1): D1100-D1106, 2017. PMID: 27924013. DOI: 10.1093/nar/gkw936

23 Tyanova S, Temu T, Sinitcyn P, Carlson A, Hein MY, Geiger T, Mann M and Cox J: The Perseus computational platform for comprehensive analysis of (prote)omics data. Nat Methods 13(9): 731-740, 2016. PMID: 27348712. DOI: 10.1038/nmeth.3901

24 Olsen JV, Blagoev B, Gnad F, Macek B, Kumar C, Mortensen P and Mann M: Global, in vivo, and site-specific phosphorylation dynamics in signaling networks. Cell 127(3): 635-648, 2006. PMID: 17081983. DOI: 10.1016/j.cell.2006.09.026

25 Plubell DL, Wilmarth PA, Zhao Y, Fenton AM, Minnier J, Reddy AP, Klimek J, Yang X, David LL and Pamir N: Extended multiplexing of tandem mass tags (TMT) labeling reveals age and high fat diet specific proteome changes in mouse epididymal adipose tissue. Mol Cell Proteomics 16(5): 873-890, 2017. PMID: 28325852. DOI: $10.1074 / \mathrm{mcp} . M 116.065524$

26 Geiger T, Wisniewski JR, Cox J, Zanivan S, Kruger M, Ishihama $\mathrm{Y}$ and Mann M: Use of stable isotope labeling by amino acids in cell culture as a spike-in standard in quantitative proteomics. Nat Protoc 6(2): 147-157, 2011. PMID: 21293456. DOI: 10.1038/nprot.2010.192

27 Shannon P, Markiel A, Ozier O, Baliga NS, Wang JT, Ramage D, Amin N, Schwikowski B and Ideker T: Cytoscape: A software 
environment for integrated models of biomolecular interaction networks. Genome Res 13(11): 2498-2504, 2003. PMID: 14597658. DOI: $10.1101 /$ gr.1239303

28 Bindea G, Mlecnik B, Hackl H, Charoentong P, Tosolini M, Kirilovsky A, Fridman WH, Pagès F, Trajanoski $\mathrm{Z}$ and Galon $\mathrm{J}$ : ClueGO: A cytoscape plug-in to decipher functionally grouped gene ontology and pathway annotation networks. Bioinformatics 25(8): 1091-1093, 2009. PMID: 19237447. DOI: 10.1093/ bioinformatics/btp 101

29 Mlecnik B, Galon J and Bindea G: Comprehensive functional analysis of large lists of genes and proteins. J Proteomics 171: 2-10, 2018. PMID: 28343001. DOI: 10.1016/j.jprot.2017.03.016

30 Kanehisa M and Goto S: KEGG: Kyoto encyclopedia of genes and genomes. Nucleic Acids Res 28(1): 27-30, 2000. PMID: 10592173. DOI: $10.1093 / \mathrm{nar} / 28.1 .27$

31 Kanehisa M, Sato Y, Kawashima M, Furumichi M and Tanabe M: KEGG as a reference resource for gene and protein annotation. Nucleic Acids Res 44(D1): D457-D462, 2016. PMID: 26476454. DOI: $10.1093 / \mathrm{nar} / \mathrm{gkv} 1070$

32 Giurgiu M, Reinhard J, Brauner B, Dunger-Kaltenbach I, Fobo G, Frishman G, Montrone C and Ruepp A: CORUM: the comprehensive resource of mammalian protein complexes-2019. Nucleic Acids Res 47(D1): D559-D563, 2019. PMID: 30357367. DOI: $10.1093 /$ nar/gky973

33 Slenter DN, Kutmon M, Hanspers K, Riutta A, Windsor J, Nunes N, Mélius J, Cirillo E, Coort SL, Digles D, Ehrhart F, Giesbertz P, Kalafati M, Martens M, Miller R, Nishida K, Rieswijk L, Waagmeester A, Eijssen LMT, Evelo CT, Pico AR and Willighagen EL: WikiPathways: A multifaceted pathway database bridging metabolomics to other omics research. Nucleic Acids Res 46(D1): D661-D667, 2018. PMID: 29136241. DOI: 10.1093/nar/gkx1064

34 Kucera M, Isserlin R, Arkhangorodsky A and Bader GD: AutoAnnotate: A cytoscape app for summarizing networks with semantic annotations. F1000Res 5: 1717, 2016. PMID: 27830058. DOI: 10.12688/f1000research.9090.1

35 Huang da W, Sherman BT and Lempicki RA: Systematic and integrative analysis of large gene lists using DAVID bioinformatics resources. Nat Protoc 4(1): 44-57, 2009. PMID: 19131956. DOI: 10.1038/nprot.2008.211

36 Krug K, Mertins P, Zhang B, Hornbeck P, Raju R, Ahmad R, Szucs M, Mundt F, Forestier D, Jane-Valbuena J, Keshishian H, Gillette MA, Tamayo P, Mesirov JP, Jaffe JD, Carr SA and Mani DR: A curated resource for phosphosite-specific signature analysis. Mol Cell Proteomics 18(3): 576-593, 2019. PMID: 30563849. DOI: $10.1074 / \mathrm{mcp}$.TIR118.000943

37 Raaijmakers LM, Giansanti P, Possik PA, Mueller J, Peeper DS, Heck AJ and Altelaar AF: PhosphoPath: Visualization of phosphosite-centric dynamics in temporal molecular networks. J Proteome Res 14(10): 4332-4341, 2015. PMID: 26317507. DOI: $10.1021 /$ acs.jproteome.5b00529

38 Darzynkiewicz Z and Juan G: DNA content measurement for DNA ploidy and cell cycle analysis. Curr Protoc Cytom Chapter 7: Unit 7.5, 2001. PMID: 18770732. DOI: 10.1002/047114 2956.cy0705s00

39 Revankar CM, Cimino DF, Sklar LA, Arterburn JB and Prossnitz ER: A transmembrane intracellular estrogen receptor mediates rapid cell signaling. Science 307(5715): 1625-1630, 2005. PMID: 15705806. DOI: 10.1126/science. 1106943

40 Dennis MK, Burai R, Ramesh C, Petrie WK, Alcon SN, Nayak TK, Bologa CG, Leitao A, Brailoiu E, Deliu E, Dun NJ, Sklar
LA, Hathaway HJ, Arterburn JB, Oprea TI and Prossnitz ER: In vivo effects of a GPR30 antagonist. Nat Chem Biol 5(6): 421427, 2009. PMID: 19430488. DOI: $10.1038 /$ nchembio. 168

41 Bologa CG, Revankar CM, Young SM, Edwards BS, Arterburn JB, Kiselyov AS, Parker MA, Tkachenko SE, Savchuck NP, Sklar LA, Oprea TI and Prossnitz ER: Virtual and biomolecular screening converge on a selective agonist for GPR30. Nat Chem Biol 2(4): 207-212, 2006. PMID: 16520733. DOI: 10.1038/nchembio775

42 Makhov PB, Golovine K, Kutikov A, Teper E, Canter DJ, Simhan J, Uzzo RG and Kolenko VM: Modulation of Akt/mTOR signaling overcomes sunitinib resistance in renal and prostate cancer cells. Mol Cancer Ther 11(7): 1510-1517, 2012. PMID: 22532600. DOI: 10.1158/1535-7163.MCT-11-0907

43 Guo H, German P, Bai S, Barnes S, Guo W, Qi X, Lou H, Liang J, Jonasch E, Mills GB and Ding Z: The PI3K/AKT pathway and renal cell carcinoma. J Genet Genomics 42(7): 343-353, 2015. PMID: 26233890. DOI: 10.1016/j.jgg.2015.03.003

44 Xia Y, Liu L, Xiong Y, Bai Q, Wang J, Xi W, Qu Y, Xu J and Guo J: Prognostic value of CC-chemokine receptor seven expression in patients with metastatic renal cell carcinoma treated with tyrosine kinase inhibitor. BMC Cancer 17(1): 70, 2017. PMID: 28114889. DOI: 10.1186/s12885-017-3065-3

45 Ito H, Ichiyanagi O, Naito S, Bilim VN, Tomita Y, Kato T, Nagaoka A and Tsuchiya N: GSK-3 directly regulates phospho4EBP1 in renal cell carcinoma cell-line: An intrinsic subcellular mechanism for resistance to mTORC1 inhibition. BMC Cancer 16: 393, 2016. PMID: 27387559. DOI: 10.1186/s12885-0162418-7

46 Marech I, Gadaleta CD and Ranieri G: Possible prognostic and therapeutic significance of c-Kit expression, mast cell count and microvessel density in renal cell carcinoma. Int J Mol Sci 15(7): 13060-13076, 2014. PMID: 25056544. DOI: 10.3390/ijms 150713060

47 Hasegawa H, Ishibashi K, Kubota S, Yamaguchi C, Yuki R, Nakajo H, Eckner R, Yamaguchi N, Yokoyama KK and Yamaguchi N: Cdk1-mediated phosphorylation of human ATF7 at Thr-51 and Thr-53 promotes cell-cycle progression into $\mathrm{M}$ phase. PLoS One 9(12): e116048, 2014. PMID: 25545367. DOI: 10.1371/journal.pone.0116048

48 Gough W, Hulkower KI, Lynch R, McGlynn P, Uhlik M, Yan L and Lee JA: A quantitative, facile, and high-throughput imagebased cell migration method is a robust alternative to the scratch assay. J Biomol Screen 16(2): 155-163, 2011. PMID: 21297103. DOI: $10.1177 / 1087057110393340$

49 Deroo BJ and Korach KS: Estrogen receptors and human disease. J Clin Invest 116(3): 561-570, 2006. PMID: 16511588. DOI: $10.1172 / \mathrm{JCI} 27987$

50 Rudalska R, Dauch D, Longerich T, McJunkin K, Wuestefeld T, Kang TW, Hohmeyer A, Pesic M, Leibold J, von Thun A, Schirmacher P, Zuber J, Weiss KH, Powers S, Malek NP, Eilers M, Sipos B, Lowe SW, Geffers R, Laufer S and Zender L: In vivo RNAi screening identifies a mechanism of sorafenib resistance in liver cancer. Nat Med 20(10): 1138-1146, 2014. PMID: 25216638. DOI: 10.1038/nm.3679

51 Li M, Wu X, Liu N, Li X, Meng F and Song S: Silencing of ATF2 inhibits growth of pancreatic cancer cells and enhances sensitivity to chemotherapy. Cell Biol Int 41(6): 599-610, 2017. PMID: 28318081. DOI: 10.1002/cbin.10760

52 Wu DS, Chen C, Wu ZJ, Liu B, Gao L, Yang Q, Chen W, Chen JM, Bao Y, Qu L and Wang LH: ATF2 predicts poor prognosis 
and promotes malignant phenotypes in renal cell carcinoma. J Exp Clin Cancer Res 35(1): 108, 2016. PMID: 27377902. DOI: 10.1186/s13046-016-0383-2

53 Hayakawa J, Mittal S, Wang Y, Korkmaz KS, Adamson E, English $\mathrm{C}$, Ohmichi M, McClelland M and Mercola D: Identification of promoters bound by c-Jun/ATF2 during rapid large-scale gene activation following genotoxic stress. Mol Cell 16(4): 521-535, 2004. PMID: 15546613. DOI: 10.1016/j.molcel.2004.10.024

54 Liu Z, Luo Q and Guo C: Bim and VDAC1 are hierarchically essential for mitochondrial ATF2 mediated cell death. Cancer Cell Int 15: 34, 2015. PMID: 25852302. DOI: 10.1186/s12935015-0188-y

55 Butz H, Ding Q, Nofech-Mozes R, Lichner Z, Ni H and Yousef GM: Elucidating mechanisms of sunitinib resistance in renal cancer: An integrated pathological-molecular analysis. Oncotarget 9(4): 4661-4674, 2017. PMID: 29435133. DOI: 10.18632/oncotarget. 23163

56 Zhou L, Liu XD, Sun M, Zhang X, German P, Bai S, Ding Z, Tannir N, Wood CG, Matin SF, Karam JA, Tamboli P, Sircar K, Rao P, Rankin EB, Laird DA, Hoang AG, Walker CL, Giaccia AJ and Jonasch E: Targeting MET and AXL overcomes resistance to sunitinib therapy in renal cell carcinoma. Oncogene 35(21): 2687-2697, 2016. PMID: 26364599. DOI: 10.1038/onc.2015.343

$57 \mathrm{Lu} \mathrm{L}, \mathrm{Li} \mathrm{Y}$, Wen H and Feng C: Overexpression of miR-15b promotes resistance to sunitinib in renal cell carcinoma. J Cancer 10(15): 3389-3396, 2019. PMID: 31293642. DOI: 10.7150/ jca.31676

58 Gao Z, Man X, Li Z, Bi J, Liu X, Li Z, Li J, Zhang Z and Kong $\mathrm{C}$ : PLK1 promotes proliferation and suppresses apoptosis of renal cell carcinoma cells by phosphorylating MCM3. Cancer Gene Ther 27(6): 412-423, 2020. PMID: 31186514. DOI: 10.1038/s41417-019-0094-X
Received February 2, 2021

Revised March 10, 2021

Accepted March 18, 2021 